\title{
Effects of Traditional Chinese Medicine Shuxuetong Injection on Random Skin Flap Survival in Rats
}

\author{
Leyi Cai, ${ }^{1}$ Wenfang Huang, ${ }^{2}$ and Dingsheng Lin ${ }^{1}$ \\ ${ }^{1}$ Department of Hand and Plastic Surgery, The Second Affiliated Hospital of Wenzhou Medical University, \\ No. 109, XueYuan Road (West), Lucheng, Wenzhou, Zhejiang 325000, China \\ ${ }^{2}$ Department of Anesthesia, The Children's Hospital Zhejiang University School of Medicine, No. 57 Zhugan Lane, \\ Hangzhou, Zhejiang 310006, China
}

Correspondence should be addressed to Dingsheng Lin; lindingsheng@gmail.com

Received 12 December 2013; Accepted 23 January 2014; Published 26 February 2014

Academic Editors: H. Acker and N. C. Gorin

Copyright (C) 2014 Leyi Cai et al. This is an open access article distributed under the Creative Commons Attribution License, which permits unrestricted use, distribution, and reproduction in any medium, provided the original work is properly cited.

Background. A Shuxuetong injection is traditionally used in Chinese medicine to treat "blood stasis and stagnation" (yu xue yu zhi). We investigated the effect of such injection on the survival of random skin flaps. Methods. McFarlane flaps were established in 60 rats divided into two groups. Postoperative celiac injections were given to both groups for 7 days. Shuxuetong was injected into the test group, and saline was injected into controls. On day 7, tissues were stained with H\&E (hematoxylin-eosin) stain, immunohistochemically evaluated, and the expression levels of xanthine oxidase were determined. Result. The mean area of flap survival in the test group was significantly higher than in controls. Expression of vascular endothelial growth factor and superoxide dismutase, and microvessel development, were markedly increased in the test group, and the malondialdehyde level was reduced. Conclusion. Shuxuetong promotes random skin flap survival.

\section{Introduction}

Local skin flaps are often created during plastic and reconstructive surgery to repair tissue defects. However, the lengthto-width ratio currently cannot exceed $1.5-2: 1$, greatly limiting the clinical applications of random skin flaps. Previous work $[1,2]$ has suggested that inadequate blood perfusion, ischemia-reperfusion injury, and expression of apoptosisrelated factors contribute directly to flap necrosis. Several pharmacological agents have been used in efforts to prevent or reverse skin flap ischemia. These include vascular endothelial growth factor (VEGF) [3], aspirin [4], and eutectic mixture of local anesthetics (EMLA; a mix of lidocaine and prilocaine) [5].

A Shuxuetong injection is traditionally used in Chinese medicine to treat "blood stasis and stagnation" (yu xue yu zhi), particularly in patients with cerebral and myocardial infarction [6]. The material is extracted from Hirudo leeches and also contains Pheretima (defined below). Hirudin, an antithrombotic substance produced by the salivary glands of the medicinal leech (Hirudo medicinalis), is the most potent and specific direct thrombin inhibitor (DTI) known [7]. Hirudin blocks the thrombin-mediated conversion of fibrinogen to fibrin, thus inhibiting clot formation. Hirudin also inhibits platelet aggregation [8]. Pheretima (from the earthworm Lumbricus bimastus) contains a thrombolytic enzyme termed lumbrokinase (PI239), which shows powerful antithrombotic and fibrinolytic activities [9]. The enzyme mediates anticoagulation by activating plasminogen, promoting thrombolysis, and preventing thrombus recurrence. Moreover, recent work has shown that Shuxuetong facilitates angiogenesis during wound healing following traumatic brain injury [6]. In addition, the material has a protective effect on brain tissue subject to ischemia-reperfusion injury, by enhancing expression of gamma-aminobutyric acid (GABA) in the hippocampus [10].

Thus, Shuxuetong injection should optimally restore the blood supply and promote wound healing. However, any utility of Shuxuetong for enhancing flap survival remains unclear. In the present work, we explored the effect of Shuxuetong on the survival of random skin flaps and the mechanism thereof. 


\section{Material and Methods}

2.1. Animal Model and Drug Administration. Sixty male Sprague-Dawley (SD) rats (250-300 g) were obtained from the Wenzhou Medical College (SCXK [Zhe] 2005-0019) and treated in accordance with the Guide for the Care and Use of Laboratory Animals of Wenzhou Medical College. The rats were randomly divided into a Shuxuetong group and a control group (30 rats/group). They were anesthetized with $5 \%$ (w/v) chloralic hydras ( $6-8 \mathrm{~mL} / \mathrm{kg}$, intraperitoneally), and then a McFarlane [11] flap $(3 \times 9 \mathrm{~cm})$ was created on the same position of the dorsum of each rat, using the iliac crest to ensure consistent positioning.

Perforated vessels at the flap bases were ligatured to create completely random vascular patterns. After separating subcutaneous tissue in the deep fascia, each flap was sutured back to the original position using continuous $4-0$ silk sutures. Prior to analysis, each flap was divided into three distinct zones of equal size, the proximal area (I), the middle area (II), and the distal area (III) [12, 13]. Aseptic techniques were rigorously applied. All rats were individually housed after surgery to prevent cannibalism or injury caused by normal socialization. All operations were performed by one researcher and no rat died during the procedure.

Commencing immediately after operation, injections of Shuxuetong (catalog number 100403-1, Mu-dan-jiang-youbo Pharmaceutical Co. Ltd., Mudanjiang, China) were given to test rats $(1.5 \mathrm{~mL} / \mathrm{kg}$; intraperitoneally for 7 successive days). Control rats received saline. At the end of treatment, flap condition was evaluated in terms of appearance, color, texture, and hair condition. Seven days later, all animals were sacrificed via administration of chloral hydrate, and flap tissues were dissected. Animal care and euthanasia conformed to the principles of the guide mentioned above.

2.2. Assessment of Survival Areas. To quantify survival area, flaps were photographed, and surviving areas were measured by superimposition of photographs on graph paper. The results are expressed as percentages of viable area, calculated as follows: extent of viable area $\times 100 /$ total area (viable and ischemic). Flap tissues were biopsied for histological assessment and immunohistochemical staining for VEGF.

2.3. Microvascular Density. Three samples $(1 \mathrm{~cm} \times 1 \mathrm{~cm})$ of central flap tissue were collected from each area and fixed in $4 \%(\mathrm{v} / \mathrm{v})$ paraformaldehyde in PBS for $24 \mathrm{~h}$. Paraffin sections were prepared using a routine technique. Slices $(4 \mu \mathrm{m})$ were subjected to $\mathrm{H} \& \mathrm{E}$ staining and examined by light microscopy (Olympus BH51, Tokyo, Japan; 100x magnification). We evaluated the thickness of granulation tissue and whether edema and neutrophil infiltration were evident. We measured microvascular density (MVD) as follows. First, we microscopically identified the most vascularized areas under low magnification (40x). Next, we counted vessels (at 400x) in five random microscopic fields each $0.152 \mathrm{~mm}^{2}$ in area, thus $0.44 \mathrm{~mm}$ in diameter. We calculated microvascularity per unit area $\left(\mathrm{mm}^{2}\right)$ as an indicator of MVD [14]. Generally, single endothelial cells, endothelial cell clusters, and vessels containing less than eight erythrocytes were considered microvessels. All slides were evaluated by two independent observers blinded to each other's findings and to experimental groupings. The mean MVD (from the two examiners) was subjected to statistical analysis.

2.4. VEGF Expression. VEGF expression level was evaluated immunohistochemically employing a streptavidin/peroxidase-based protocol. We first blocked slides with normal goat serum at room temperature for $20 \mathrm{~min}$ and added $50 \mu \mathrm{L}$ antiVEGF antibody solution (diluted 1:100). Incubation at $4^{\circ} \mathrm{C}$ overnight followed. All slices were warmed to $37^{\circ} \mathrm{C}$ for $45 \mathrm{~min}$ and washed with PBS. Next, $50 \mu \mathrm{L}$ goat anti-rat antibody (diluted 1:50) was added, followed by incubation at $37^{\circ} \mathrm{C}$ for $1 \mathrm{~h}$ and rinsing with PBS. For color development, samples were incubated in 3,3 N-diaminobenzidine tetrahydrochloride (DAB) solution for $5 \mathrm{~min}$. The most intensely stained areas were identified under low magnification, and then vessels in five fields of each slice were viewed under higher magnification (400x). Observation parameters (white balance, aperture, shutter speed, and time) were held constant. Images were saved using Image-Pro Plus software, version 6.0 (Media Cybernetics, Rockville, MD) and the integral absorbance (IA) values were used as indicators of VEGF expression levels.

2.5. Analysis of Superoxide Dismutase Activity and Evaluation of Malondialdehyde Content. On day 7 postoperatively, 30 tissue specimens $(0.5 \mathrm{~cm} \times 0.5 \mathrm{~cm})$ were obtained from the section II/III boundaries, weighed, homogenized, and diluted to $10 \%(\mathrm{v} / \mathrm{v})$ in an ice bath. Superoxide dismutase (SOD) activity was determined using the xanthine oxidase method, and malondialdehyde (MDA) content was measured by reacting the material with thiobarbituric acid (TBA) at $90-$ $100^{\circ} \mathrm{C}[15]$.

2.6. Statistical Analysis. All results are expressed as means \pm SDs. Data were analyzed with the aid of SPSS version 16.0 software. Graphs were constructed using GraphPad Prism version 5.0.

A $P$ value $<0.05$ was considered statistically significant. The extent of necrotic change and histological and immunohistochemical findings were compared using the MannWhitney test.

\section{Results}

3.1. Shuxuetong Improves Flap Survival. The general condition of each flap was recorded daily at the time of injection. On the first day, all flaps were swollen to some extent, and distal area III was dark purple in color, but without obvious necrosis. On the third day, flap areas II and III in both control and experimental groups exhibited reddish-brown focal or patchy necrosis, with congestion. On the seventh day, most of these necrotic parts had fused, scabbed, and hardened. The boundaries between necrotic and surviving regions were stable. Surviving flap portions grew fine hair but the necrotic regions became hard, dark, and glabrous and did not bleed when cut with a scalpel (Figure 1). 

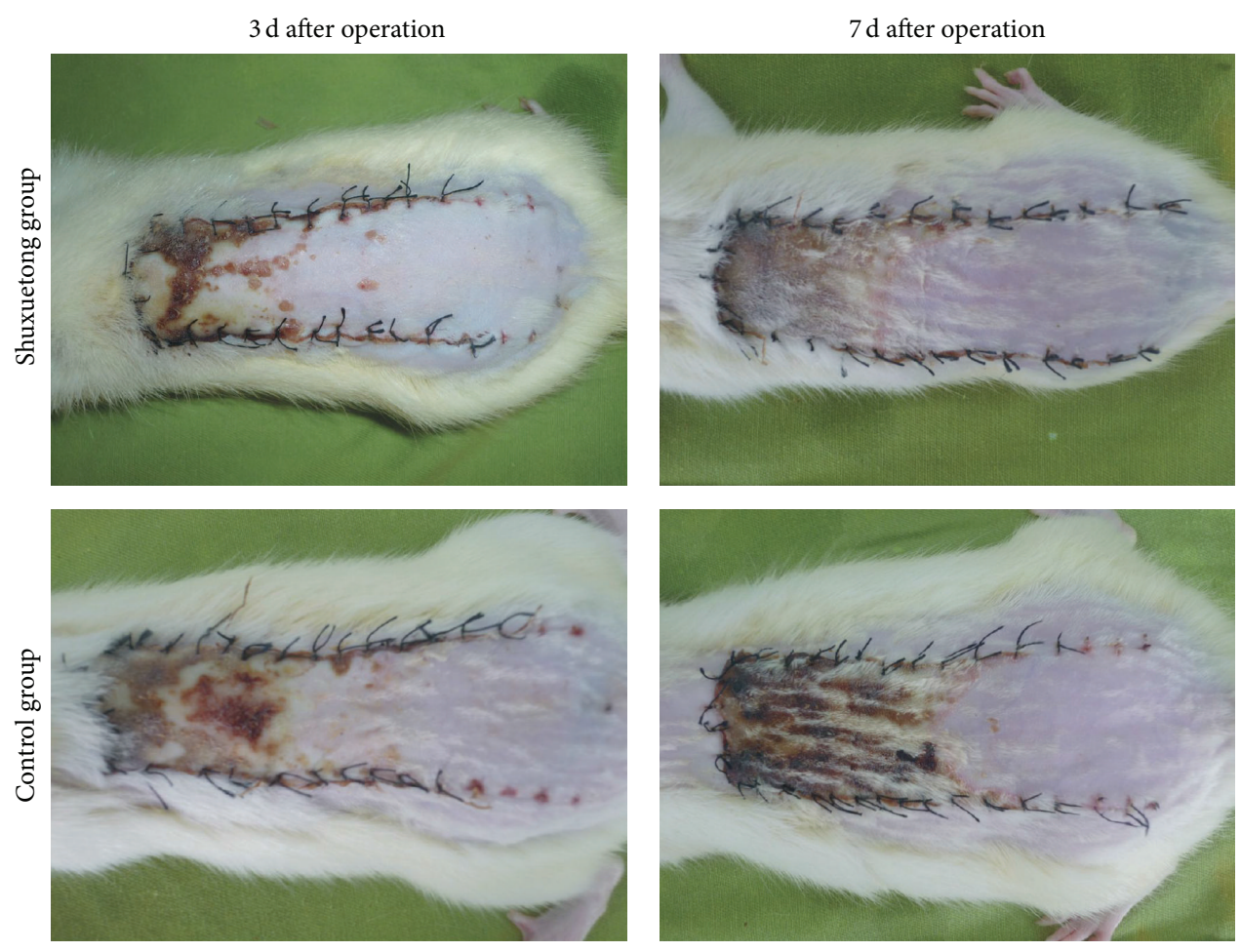

FIGURE 1: Digital photographs showing the general form of postoperative flaps in the Shuxuetong and control groups. The photographs were taken on days 3 and 7.

The mean surviving area was $72.52 \pm 2.23 \%$ in the Shuxuetong group, significantly higher than the $50.36 \pm$ $2.37 \%$ in the control group $(P<0.05)$.

3.2. Shuxuetong Facilitates Angiogenesis. Seven days after operation, the distal areas were morphologically similar in histological terms. All flaps exhibited similar changes in appearance; inflammatory cell infiltration was prominent, as were structural damage and edema. Ninety percent of tissue images revealed degeneration and necrosis of muscle fibers. In flap area II, the Shuxuetong group exhibited greater proliferation of fibroblasts, thinner granulation tissue with less edema, more scattered areas of subcutaneous hemorrhage, more diffuse neutrophil infiltration, and more neovascularization, compared to the control group (Figure 2). In area I, the Shuxuetong group exhibited edema, vascular dilation, and inflammatory cell infiltration to a lesser extent than controls.

The MVDs of area I in the Shuxuetong and control groups were $34.71 \pm 6.37 / \mathrm{mm}^{2}$ and $32.71 \pm 5.36 / \mathrm{mm}^{2}$, respectively, (Figure 3$)$, thus not significantly different $(P>0.05)$. The respective MVDs of area II were $27.42 \pm 4.21 / \mathrm{mm}^{2}$ and $17.45 \pm 5.43 / \mathrm{mm}^{2}$ (Figure 3 ). This difference was statistically significant $(P<0.05)$.

3.3. VEGF Expression by Endothelial Cells Is Enhanced by Shuxuetong. A significant $(P<0.05)$ difference in VEGF expression was evident between the two groups as revealed by IA data (Figure 4). The expression level in the Shuxuetong group was 4,731.24 \pm 448.99 IA and that of the control group was $2,466.01 \pm 801.67$.
3.4. Shuxuetong Protects Flap against Ischemia-Reperfusion Injury. The mean SOD activity in the Shuxuetong group was $54.633 \pm 2.497$ units/mg protein, significantly higher than that in the control group (37.34 $\pm 8.842 ; P<0.05)$ (Figure 5). However, the mean MDA level in the experimental group $(23.028 \pm 9.357 \mathrm{nmol} / \mathrm{mg}$ protein) was significantly less than that in the control group $(58.810 \pm 10.290)(P<0.05)$ (Figure 6).

\section{Discussion}

The components of Shuxuetong are derived from the leech and the earthworm. In Chinese traditional medicine, both components promote blood flow (huo xue), activate meridians (tong luo), and disperse blood stasis (hua yu) [6]. Thus, we logically hypothesized that Shuxuetong might facilitate angiogenesis.

The role played by VEGF in initiating proliferation of endothelial cells has been well documented. VEGF promotes revascularization of ischemic flaps, thus increasing flap survival $[16,17]$. Moreover, Shuxuetong injections enhance VEGF/VEGFR-2 expression in patients with traumatic brain injuries $[6,18]$. In the present study, the VEGF expression level was markedly higher in test flaps than in controls. Furthermore, the MVD in area II of the Shuxuetong group was significantly greater than that in controls. These results suggest that Shuxuetong may promote neovascularization and microcirculation in ischemic flaps by increasing VEGF expression, ultimately improving flap viability. The detailed mechanism requires further study. 

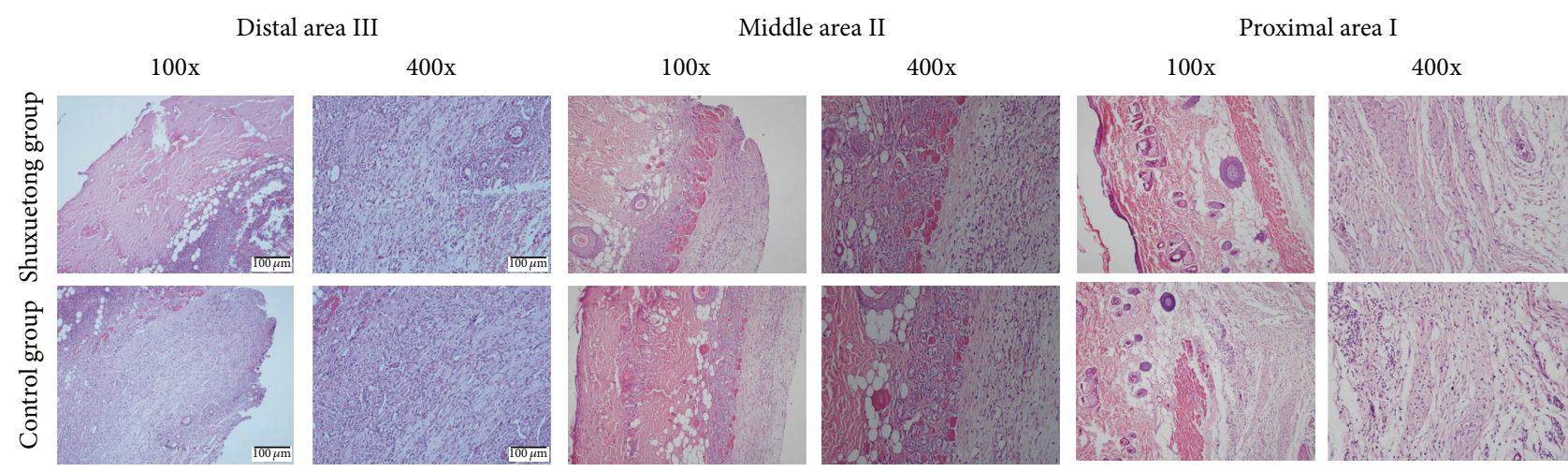

FIGURE 2: Histological changes in areas I, II, and III of flaps of the Shuxuetong and control groups. Magnifications: 100x and 400x. H\&E stain.

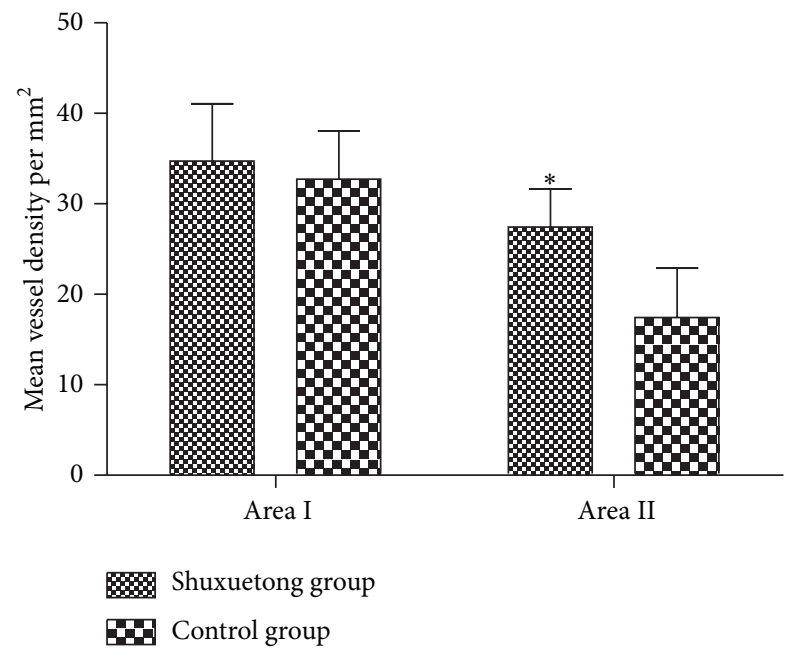

Figure 3: The MVDs of areas I and II of the ShuXueTong and control groups.

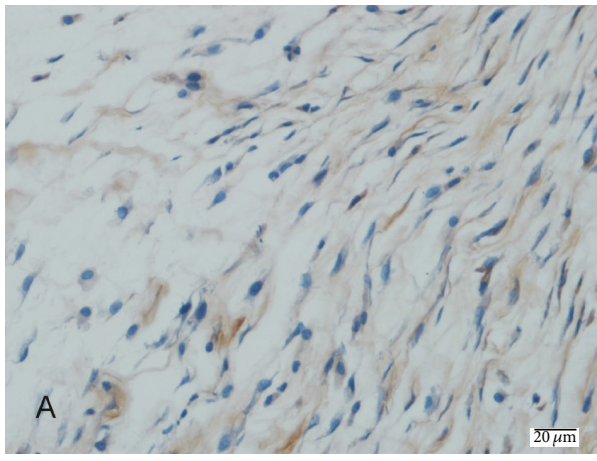

(a) Shuxuetong group

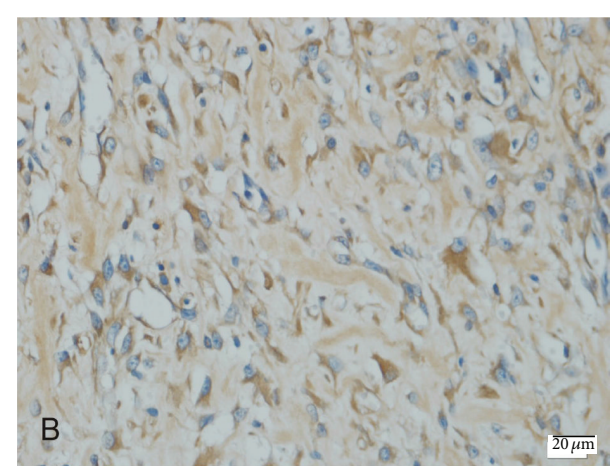

(b) Control group

FIGURE 4: VEGF protein levels in the Shuxuetong and control groups. (immunohistochemical staining) $(\times 400)$.

Generation of oxygen-derived free radicals contributes greatly to the pathogenesis of ischemia-reperfusion injury, and the topic has been well studied. A burst of free oxygen radicals attacks lipids of the cell membrane as well as membrane proteins within the first few minutes of reperfusion. In addition, reperfusion leads to the accumulation of activated neutrophils in ischemic tissue and activation of xanthine oxidase in endothelial cells, causing rapid necrosis of the flap $[15,19]$.

SOD, metalloprotein, is an important antioxidase that inactivates $\mathrm{O}^{-2}$ (the predecessor of $\mathrm{H}_{2} \mathrm{O}_{2}$ and $\mathrm{OH}^{-}$), thus protecting cells from injury by toxic oxygen-derived free radicals. Such radicals cause peroxidation of lipids and proteins and damage cell and organelle membranes, thus destroying 


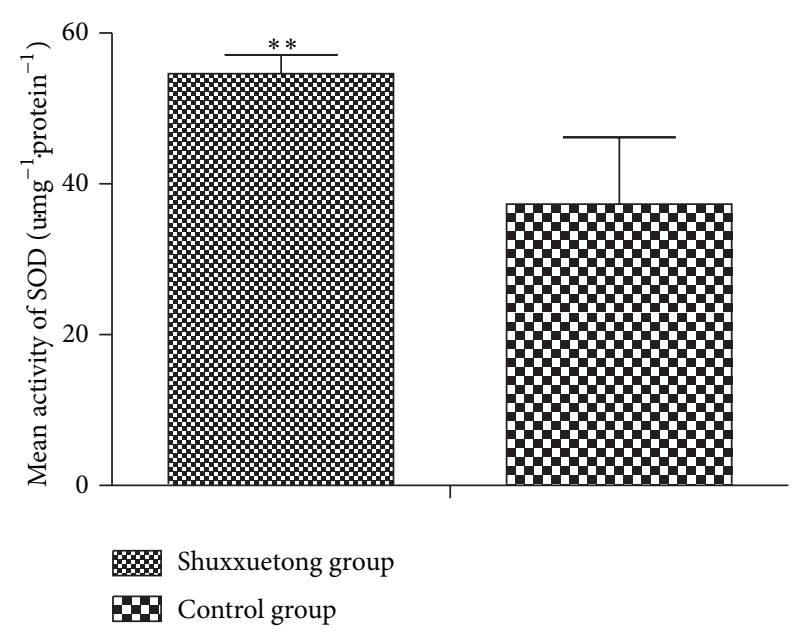

FIGURE 5: SOD activities in the Shuxuetong and control groups.

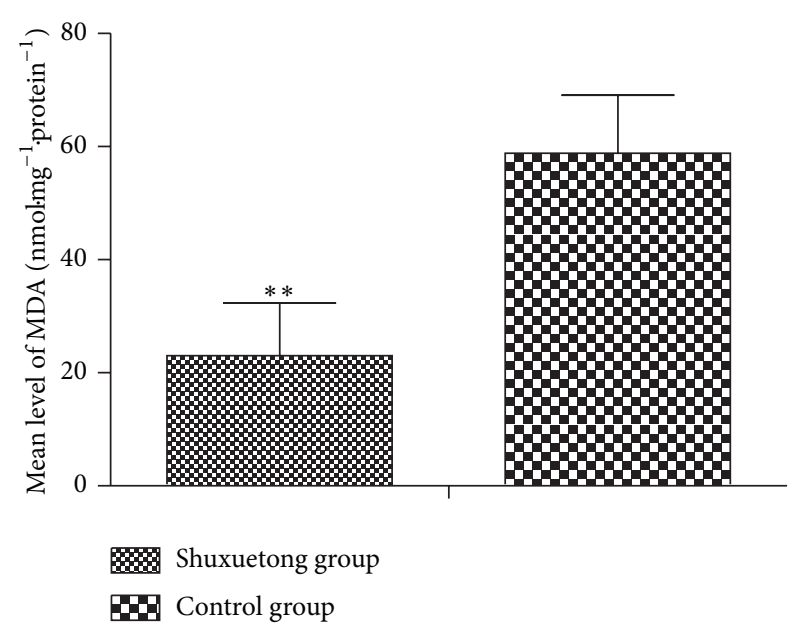

FIGURE 6: MDA levels in the Shuxuetong and control groups.

tissue structure and function. MDA is one product of lipid peroxidation, and the MDA level thus indirectly reflects the extent of tissue damage [20]. In the present study, we found that SOD activity, MDA level, and the surviving flap area were significantly better in the test group than in controls. We thus confirmed that Shuxuetong had a protective effect in terms of endogenous SOD activity and inhibited lipid peroxidation. It also inhibited the development of ischemic reperfusion injury by increasing free radical scavenging.

The antithrombotic, anticoagulant, and fibrinolytic activities of Shuxuetong may also contribute to the survival of random skin flaps.

\section{Conclusions}

Shuxuetong injection should be considered potentially as a choice for microsurgery. Undoubtedly, this medicine shows an excellent result for the survival of skin random flap.

\section{Conflict of Interests}

The authors have any conflict of interests.

\section{Acknowledgments}

The authors would like to express their sincere thanks to the financial support: Zhejiang Provincial Association of Integrative Medicine Clinical Pharmacy Research (Grant no. 2012LY015) and 2012 College Students of Science and Technology Innovation Program of Zhejiang Province (Grant no. 2012R413032).

\section{References}

[1] G. Jin and S. Zhang, "Function of apoptosis-related factors on surgical flap survival," Journal of North China Coal Medical College, vol. 8, pp. 313-315, 2006.

[2] M. Yang, L. Sheng, H. Li, R. Weng, and Q.-F. Li, "Improvement of the skin flap survival with the bone marrow-derived mononuclear cells transplantation in a rat model," Microsurgery, vol. 30, no. 4, pp. 275-281, 2010.

[3] Y. Pang, W. C. Lineaweaver, M.-P. Lei et al., "Evaluation of the mechanism of vascular endothelial growth factor improvement of ischemic flap survival in rats," Plastic and Reconstructive Surgery, vol. 112, no. 2, pp. 556-564, 2003.

[4] A. Shalom, T. Friedman, and M. Westreich, "Effect of aspirin and heparin on random skin flap survival in rats," Dermatologic Surgery, vol. 34, no. 6, pp. 785-790, 2008.

[5] N. Karaçal, Ö. Ambarcioğlu, U. Topal, T. Mamedov, and N. Kutlu, "Enhancement of dorsal random-pattern skin flap survival in rats with topical lidocaine and prilocaine (EMLA): enhancement of flap survival by EMLA," Journal of Surgical Research, vol. 124, no. 1, pp. 134-138, 2005.

[6] X. Jin, G. Shen, F. Gao et al., "Traditional Chinese drug ShuXueTong facilitates angiogenesis during wound healing following traumatic brain injury," Journal of Ethnopharmacology, vol. 117, no. 3, pp. 473-477, 2008.

[7] Y. Huang, Y. Zhang, Y. Wu et al., "Expression, purification, and mass spectrometric analysis of ${ }^{15} \mathrm{~N},{ }^{13} \mathrm{C}$-labeled RGD-hirudin, expressed in Pichia pastoris, for NMR studies," PLOS ONE, vol. 7, no. 8, Article ID e42207, 2012.

[8] W. Mo, Y.-L. Zhang, H.-S. Chen, L.-S. Wang, and H.-Y. Song, "A novel hirudin derivative characterized with anti-platelet aggregations and thrombin inhibition," Journal of Thrombosis and Thrombolysis, vol. 28, no. 2, pp. 230-237, 2009.

[9] T. Ge, Z.-J. Sun, S.-H. Fu, and G.-D. Liang, "Cloning of thrombolytic enzyme (lumbrokinase) from earthworm and its expression in the yeast Pichia pastoris," Protein Expression and Purification, vol. 42, no. 1, pp. 20-28, 2005.

[10] D.-J. Zhang, G.-R. Xu, Z.-Y. Li et al., "The effects of Shuxuetong on the pathology of cerebral ischemia-reperfusion injury and GABA and TNF- $\alpha$ expression in gerbil models," Neuroscience Bulletin, vol. 22, no. 1, pp. 41-46, 2006.

[11] S. J. Mandriota, C. Pyke, C. Di Sanza, P. Quinodoz, B. Pittet, and M. S. Pepper, "Hypoxia-inducible angiopoietin-2 expression is mimicked by iodonium compounds and occurs in the rat brain and skin in response to systemic hypoxia and tissue ischemia," The American Journal of Pathology, vol. 156, no. 6, pp. 20772089, 2000.

[12] C. P. Kelly, A. Gupta, M. Keskin, and I. T. Jackson, "A new design of a dorsal flap in the rat to study skin necrosis and its prevention," Journal of Plastic, Reconstructive and Aesthetic Surgery, vol. 63, no. 9, pp. 1553-1556, 2010. 
[13] T. Xiao-xiao, W. Sen-Min, and L. Ding-sheng, "Effects of vinpocetine on random skin flap survival in rats," Journal of Reconstructive Microsurgery, vol. 29, no. 6, pp. 393-398, 2013.

[14] Z. Chen, T. Wang, L. Cai et al., "Clinicopathological significance of non-small cell lung cancer with high prevalence of Oct4 tumor cells," Journal of Experimental and Clinical Cancer Research, vol. 31, no. 1, article 10, 2012.

[15] F. Ozkan, Y. Şenayli, H. Ozyurt, U. Erkorkmaz, and B. Bostan, "Antioxidant effects of propofol on tourniquet-induced ischemia-reperfusion injury: an experimental study," Journal of Surgical Research, vol. 176, no. 2, pp. 601-607, 2012.

[16] F. Zhang, K. Fischer, E. Komorowska-Timek et al., "Improvement of skin paddle survival by application of vascular endothelial growth factor in a rat TRAM flap model," Annals of Plastic Surgery, vol. 46, no. 3, pp. 314-319, 2001.

[17] F. Zhang and W. Lineaweaver, "Acute and sustained effects of vascular endothelial growth factor on survival of flaps and skin grafts," Annals of Plastic Surgery, vol. 66, no. 5, pp. 581-582, 2011.

[18] J. Wei, B. Chen, and Z. Tian, "Effect of Shuxuetong Injection on VEGF expression of hind-limb ischemia vascular disorder in diabetic rats," Chinese Journal of Integrative Medicine on CardioCerebrovascular Disease, vol. 7, pp. 1048-1049, 2009.

[19] J. Cui, L. Liu, J. Zou et al., "Protective effect of endogenous hydrogen sulfide against oxidative stress in gastric ischemiareperfusion injury," Experimental and Therapeutic Medicine, vol. 5, no. 3, pp. 689-694, 2013.

[20] L. Sun, Y. Li, J. Shi, X. Wang, and X. Wang, "Protective effects of ligustrazine on ischemia-reperfusion injury in rat kidneys," Microsurgery, vol. 22, no. 8, pp. 343-346, 2002. 


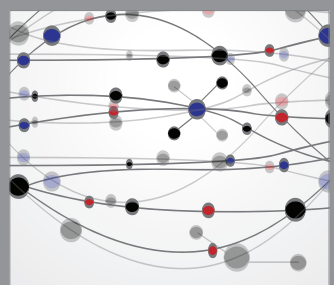

The Scientific World Journal
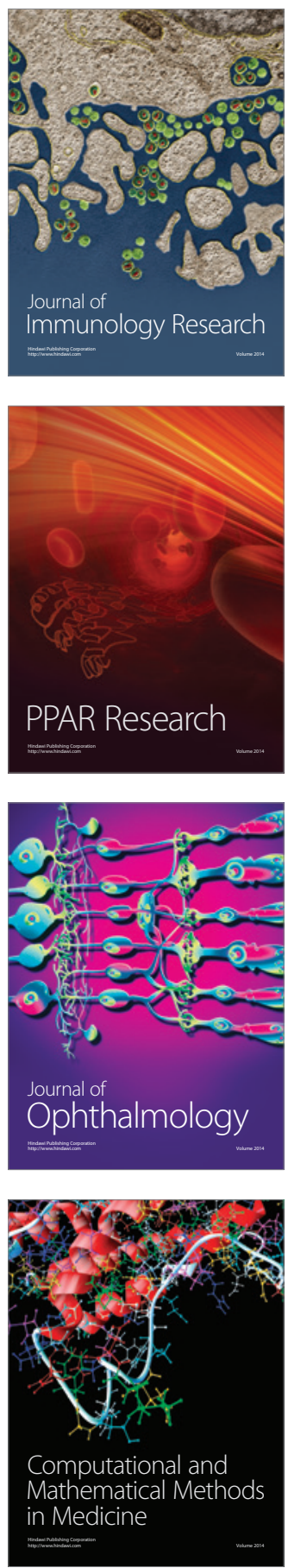

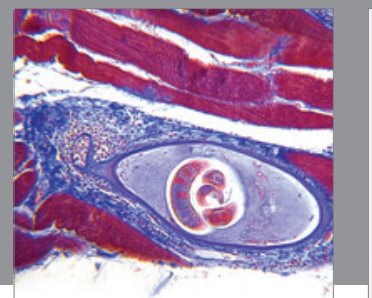

Gastroenterology

Research and Practice
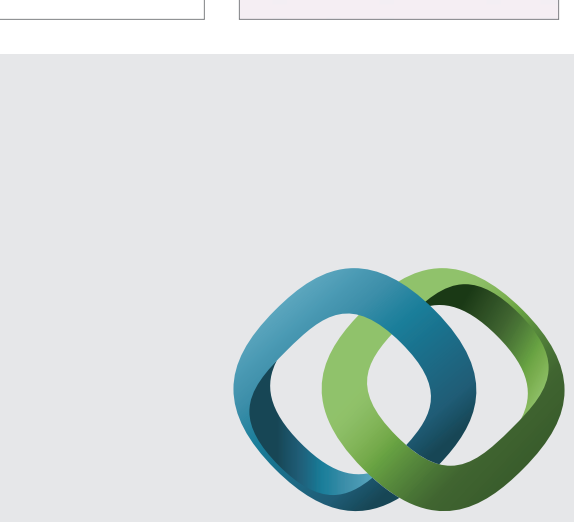

\section{Hindawi}

Submit your manuscripts at

http://www.hindawi.com
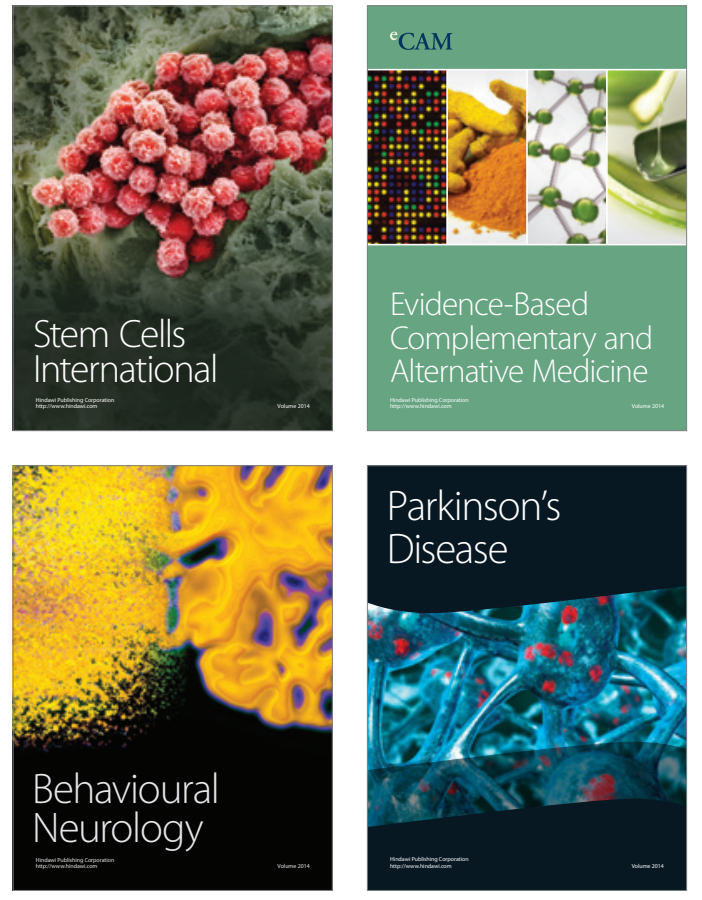
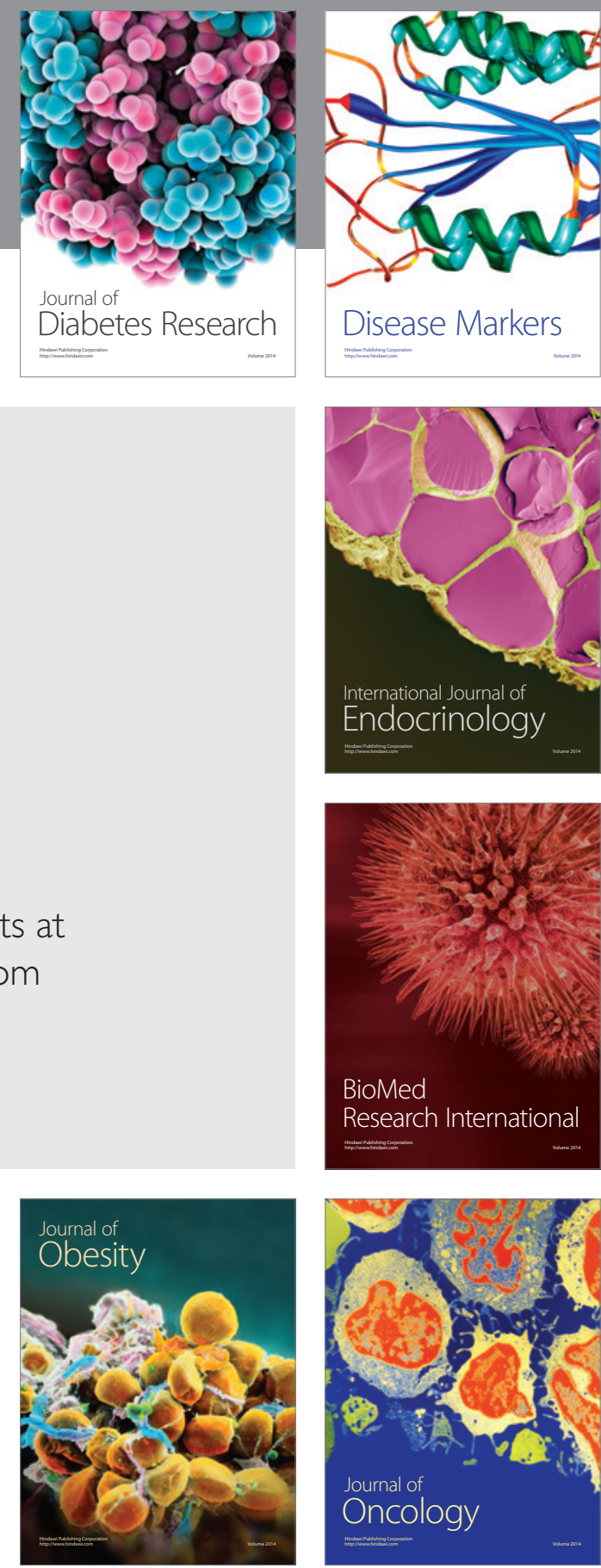

Disease Markers
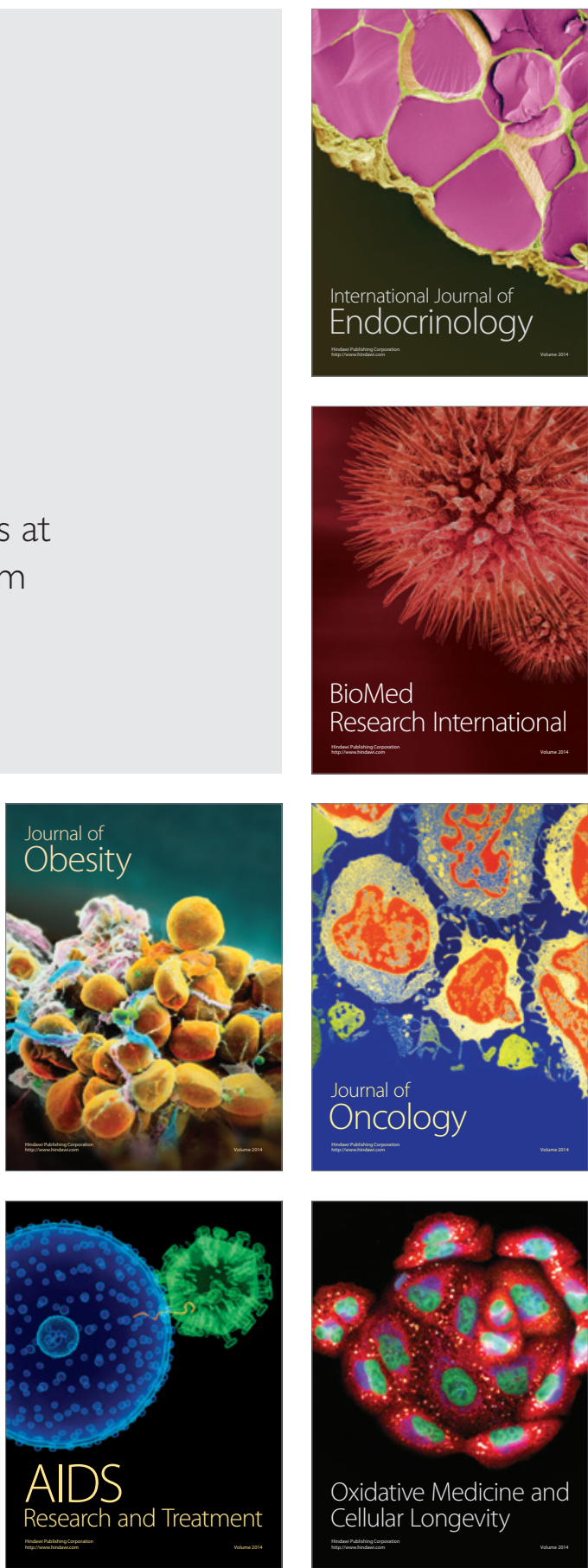